\title{
Nanoimaging using soft X-ray and EUV laser-plasma sources
}

\author{
Przemyslaw Wachulak ${ }^{1, *}$, Alfio Torrisi ${ }^{1}$, Mesfin Ayele ${ }^{1}$, Andrzej Bartnik ${ }^{1}$, Joanna Czwartos ${ }^{1}$, Eukasz Węgrzyński ${ }^{1}$, \\ Tomasz Fok ${ }^{1}$ and Henryk Fiedorowicz ${ }^{1}$ \\ ${ }^{1}$ Institute of Optoelectronics, Military University of Technology, 2, Kaliskiego Street, 00-908 Warsaw, Poland
}

\begin{abstract}
In this work we present three experimental, compact desk-top imaging systems: SXR and EUV full field microscopes and the SXR contact microscope. The systems are based on laser-plasma EUV and SXR sources based on a double stream gas puff target. The EUV and SXR full field microscopes, operating at $13.8 \mathrm{~nm}$ and $2.88 \mathrm{~nm}$ wavelengths are capable of imaging nanostructures with a sub-50 nm spatial resolution and short (seconds) exposure times. The SXR contact microscope operates in the "waterwindow" spectral range and produces an imprint of the internal structure of the imaged sample in a thin layer of SXR sensitive photoresist. Applications of such desk-top EUV and SXR microscopes, mostly for biological samples (CT26 fibroblast cells and Keratinocytes) are also presented. Details about the sources, the microscopes as well as the imaging results for various objects will be presented and discussed. The development of such compact imaging systems may be important to the new research related to biological, material science and nanotechnology applications.
\end{abstract}

\section{Introduction}

Use Recent advancements in nanoscience and nanotechnology will surely not be possible without nanometer scale resolution imaging tools and techniques, such as extreme ultraviolet (EUV) and soft X-ray (SXR) [1] microscopy. The EUV/SXR microscopy proved to be a useful tool for imaging of objects with nanometer spatial resolution and allows to obtain additional information about the objects investigated, providing at the same time high optical contrast in the specific wavelength ranges. The EUV radiation is strongly absorbed in thin layers of materials, so it is highly suitable for investigations of thin films and layers of materials, while the SXR radiation, specifically in the "water-window" $(\lambda=2.3-4.4 \mathrm{~nm})$, is particularly suitable for high resolution biological imaging, due to high achievable contrast between carbon and water - the main constituents of biological material.

Most of studies in this field are conducted using large scale facilities, such as synchrotron or free-electron laser installations [2,3]. Synchrotron and FEL facilities are being used for cutting-edge scientific experiments and provide highest available photon flux, tunability and spatial and temporal coherence. However, they also have some disadvantages (high maintenance cost, require highly trained staff due to the complexity of those sources and have limited user access). Recent progress in development of a new generation of compact EUV and SXR compact sources, especially laser-plasma sources permits to overcome some of these limitations and allow for imaging experiments in the laboratories worldwide. So far, many efforts have been made to perform nanometer spatial resolution imaging in the EUV and
SXR spectral ranges over the last few years employing both large scale and compact sources. Some examples of such works are listed as follows. The synchrotron radiation at $\lambda=2.4 \mathrm{~nm}$ was used for imaging frozenhydrated samples at atmospheric pressure [4] to observe internal details of algae cells with a spatial resolution of $\sim 35 \mathrm{~nm}$. A SXR source, emitting at $\lambda=2.88 \mathrm{~nm}$, based on a liquid jet nitrogen target was employed recently for microscopy in the "water-window" range with a sub-50 $\mathrm{nm}$ spatial resolution, but this system present a very complicated hardware [5]. A compact high order harmonic generation (HHG) source was employed for sub-100 nm spatial resolution imaging [6]. However, the HHG systems require a femtosecond laser as a driver for HHG process with conversion efficiency ( 10-6-10-4) which often results in long exposures or does not allow a proper reconstruction of the image. Ptychographic techniques, usually based on employment of hard X-ray beams, although provide very high spatial resolution, are extensively time consuming during the reconstruction process [7]. Imaging in the EUV range permits to analyse very thin samples, nanofilms and nanostructures, because the EUV radiation is absorbed by solid materials with thicknesses of the order of $100 \mathrm{~nm}$ [8] and by gaseous materials with thicknesses of the order of a few millimetres [9]. Employing such radiation allows to visualize in a direct way through absorption contrast mechanism the flow of the gas, usually investigated with other techniques, such as the interferometry [10]. It was demonstrated that the radiation from a capillary discharge laser operating at a wavelength of $=46.9 \mathrm{~nm}$ EUV images permits to obtain a spatial resolution better than $55 \mathrm{~nm}[11,12]$, and that the spatial resolution of holographic images [13], employing the same

Corresponding author: : wachulak@gmail.com 
wavelength, can be improved up to sub-50 nm [14]. A table top EUV laser emitting $13.2 \mathrm{~nm}$ wavelength radiation from Ni-like $\mathrm{Cd}$ ions was employed for imaging of with a sub-38 nm spatial resolution and a picosecond time resolution [15]. Using xenon gas discharge source a sub-100 $\mathrm{nm}$ zone-plate based zooming EUV microscope was demonstrated [16]. Very nice results were also recently demonstrated [17] with liquid nitrogen target based system and $1.3 \mathrm{kHz}$ repetition rate $\mathrm{Nd}$ Y YAG laser to record images of biological samples and nanostructures with half-pitch spatial resolution of $40-50 \mathrm{~nm}$, however, the laser driver was quite complicated and large comparing to other, more compact ones. Another interesting and already well established technique for obtaining high resolution images of samples is contact microscopy. It uses the SXR radiation transmitted through the sample to expose a high resolution photoresist in contact with the sample. Using this method human platelets [18], fibroblasts [19] and hydrated biological cells [20] were imaged.

It is necessary, however, to find a good compromise in the desk-top SXR/EUV imaging systems, between the performance (high spatial resolution, low exposure time provided by large scale facilities) and the complexity, size, cost of these setups. Some of these problems can be addressed employing laser plasma sources based on double stream gas puff target for microscopy purposes. These sources represent a useful alternative for lab-scale analysis in order to perform high spatial resolution imaging by using short wavelength radiations.

In this article we would like to show our recent developments of three simple and compact SXR/EUV microscopes, capable of resolving 50-80 $\mathrm{nm}$ features that require short exposure times and have a desk-top footprint. The laser-plasma source employed allows the possibility to generate the plasma efficiently and without debris production, they are also robust and easy to operate, providing relatively high EUV and SXR emission flux [21]. Additionally, they have compact and easy construction. Moreover, the EUV/SXR microscopes, based on those sources [22] do not require sample preparation such as gold coating for SEM microscopes for example or marking/staining the samples for STED microscopy. In such systems the gas puff target is produced by injection of a small amount of high-Z gas - working gas, into a stream of low-Z gas outer gas, by a fast electromagnetic double valve system, as depicted in Fig. 1a). The gas puff target is then irradiated by focused laser pulses from Nd:YAG laser, which allows generation of the EUV and SXR radiation. Both full-field microscopes use reflective optics to focus the short wavelength radiation onto a sample and FZPs to obtain magnified images of the sample with high spatial resolution, while the contact microscope uses the radiation directly, without pre-focusing, to form an imprint of the sample in the photoresist layer.

\section{Microscopy systems}

Soft X-ray contact microscope and EUV/SXR microscopes based on Fresnel optics are schematically depicted in Fig. 1b) and Fig. 1c), respectively. The sources for those microscopes were developed using a compact Nd:YAG laser, $\lambda=1064 \mathrm{~nm}, 0.5-0.7 \mathrm{~J}$ pulse energy, $3 \mathrm{~ns}$ pulse duration and $1-10 \mathrm{~Hz}$ repetition rate.

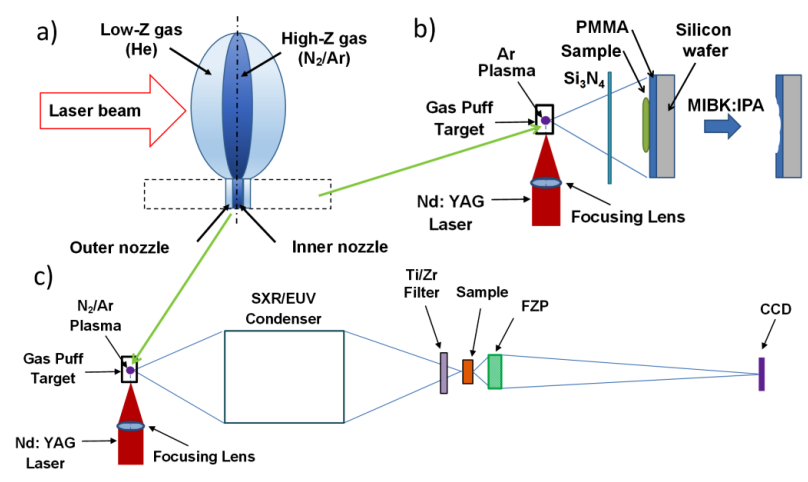

Fig. 1. a) Scheme of the double stream gas puff target employed in laser-plasma EUV/SXR sources, b) scheme of the contact SXR microscope and c) general scheme of the SXR/EUV full-field microscopes.

The laser beam is focused onto a double-stream gas puff target, schematically depicted in Fig. 1a), produced by an electromagnetic valve with double radially-symmetric nozzles. Laser pulses irradiate the gaseous target producing plasma, which efficiently emits radiation from various spectral ranges, including the EUV and SXR regions of electromagnetic spectrum. The gas puff target source was optimized for emissions in the "water window" (both quasi-monochromatic for the SXR fullfield microscope and broadband for contact SXR microscope) and the EUV spectral ranges using spectral narrowing with thin filters, depending on the type of the microscope. A nitrogen plasma emission, filtered with $\mathrm{Ti}$ foil, provides $\lambda=2.88 \mathrm{~nm}$ wavelength radiation and an $\mathrm{Ar}$ plasma emission, filtered with $\mathrm{Mo} / \mathrm{Si}$ multilayer mirror and $\mathrm{Zr}$ filter, provides radiation at $\lambda=13.84 \mathrm{~nm}$. However, for the argon/helium plasma if silicon nitride filter is used, it provides emission from $\lambda=2.8-4 \mathrm{~nm}$, well within the "water window" spectral range with much larger number of photons, suitable for contact microscopy. Such sources were employed for SXR and EUV experiments, respectively. The gas pressures, nozzle position in respect to the laser focus, valve timing in respect to the laser pulse were optimized in order to maximize the photon flux at the sample plane.

The inner nozzle injects a small amount of working gas (high $\mathrm{Z}$ gas $-\mathrm{N}_{2}$ at 8 bar, in case of SXR full-field microscope, and Ar, at 10 bar, in case of the EUV fullfield microscope and contact SXR microscope), while the outer nozzle injects an outer gas (low Z gas, He, with a pressure of 6 bar), to narrow down the flow of the working gas, reducing its density gradient along the nozzle axis. This allows obtaining higher target density at 1-2 $\mathrm{mm}$ from the nozzle exit and, in turn, allows for higher photon yield in the EUV/SXR region. Interaction of Nd:YAG laser pulses with gaseous target produces 
plasma. The radiation from the plasma is focused using condenser optics and illuminates the sample, located in a second focal point of a condenser in the case of full-field microscopes. Depending of the type of microscope, plasma radiation is collected and focused by a different condenser and filter, and images of the objects are obtained with different zone plates using a backilluminated CCD camera. In the case of contact SXR microscope the scheme is much simpler, because the radiation from the plasma is transmitted through a 200 $\mathrm{nm}$ thick $\mathrm{Si}_{3} \mathrm{~N}_{4}$ filter and illuminated the sample directly, to form its imprint in the surface of the photoresist. In the following paragraphs the systems will be described in more detail.

In the full field SXR microscope to focus the SXR radiation from the nitrogen plasma an ellipsoidal, axisymmetrical SXR condenser (Rigaku, Czech Republic) coated with nickel film was used. A titanium filter (200 nm thick, Lebow), positioned downstream the condenser, selects the He-like nitrogen line at $\lambda=2.88 \mathrm{~nm}$ from nitrogen plasma emission, suitable for the objective optic. Filtered SXR radiation illuminates the sample/object, positioned $140 \mathrm{~mm}$ downstream the condenser, in the second focal point. A FZP objective (Zoneplates Ltd., UK) was used to form a magnified image onto a back-illuminated SXR-sensitive CCD camera (Andor, iKon-M DO-934-BN, 1Mpixel, 13 x 13 $\mu \mathrm{m}^{2}$ pixel size) in transmission mode. A silicon nitride FZP (400 nm thick, $250 \mu \mathrm{m}$ in diameter, outer zone width of $30 \mathrm{~nm}$ ) with a focal length $\mathrm{f}=2.6 \mathrm{~mm}$ at 2.88 nm wavelength, was used as an objective. The numerical aperture of the zone plate was matched to the numerical aperture of the condenser, providing incoherent illumination [23]. The geometrical magnification of the system was $\sim 220 x$. It was already demonstrated that such system is capable to achieve a half-pitch spatial resolution of $\sim 60 \mathrm{~nm}$ [24]. More details about this work can be found in [25].

In the full field EUV microscope an Ar plasma radiation was collected and spectrally narrowed by an ellipsoidal off-axis mirror coated with a $\mathrm{Mo} / \mathrm{Si}$ multilayer (MLM), employed as a condenser (Reflex, Czech Republic, and IOF, Germany). The condenser was designed and manufactured in order to reflect the radiation at the wavelength of $13.5 \pm 0.5 \mathrm{~nm}$ at $45^{\circ}$ incidence angle. To eliminate longer wavelengths, emitted from the Ar plasma (at wavelengths $\lambda 16 \mathrm{~nm}$ ), a $250 \mathrm{~nm}$ thick zirconium filter (Lebow) was used. The sample was imaged using a FZP objective (diameter 200 $\mu \mathrm{m}$, number of zones 1000 and outer zone width $\Delta \mathrm{r}=50$ nm, made from PMMA, from Zoneplates Ltd., UK) onto the CCD camera (Andor, iKon-M DO-934-BN camera). The FZP was fabricated using e-beam lithography in a $200 \mathrm{~nm}$ thick PMMA layer deposited on top of a $50 \mathrm{~nm}$ thick Si3N4 membrane. As in case of the SXR microscope, an incoherent illumination was provided by matching of the numerical aperture of the condenser and of the FZP objective. The geometrical magnification of the objective was $410 \mathrm{x}$, however, it can be easily changed by adjusting the FZP-CCD distance. Such system, in this configuration, is capable to achieve a half-pitch spatial resolution of $48 \mathrm{~nm}$ [26]. More details about this system can be found in [27].

In the contact SXR microscope the Ar plasma emission was narrowed down to the "water-window" spectral range by employing $200 \mathrm{~nm}$ thick Si3N4 filter. Using that filter the most energy will reside in the wavelength range from $2.8 \mathrm{~nm}$ to $4 \mathrm{~nm}$, well within the "water-window" range. Such broad band SXR radiation from $\mathrm{Ar} / \mathrm{He}$ gas puff target has approximately one order of magnitude more photons than from N2/He gas puff target, which allows in this case for irradiation and exposure of the high resolution photoresist (500 nm thick PMMA on top of a silicon wafer). The object is then placed in contact with the PMMA. The light that is locally transmitted by the object's structure illuminates the photoresist and changes its physical and chemical structure. After the irradiation the photoresist is chemically developed in solution of methyl isobutyl ketone and isopropyl alcohol (MIBK:IPA 1:2 v/v) for 90 seconds and modulation of the light intensity absorbed by the object is converted in this process to a modulation of the thickness of the resist. This creates a relief-like structure in its surface. The height of the relief structure is directly proportional (however, not necessarily linearly), to the dose of radiation absorbed in the resist volume. In such process a high resolution imprint of the internal structure of the object can be stored in the nearsurface of the photoresist and later converted to an image using for example AFM or SEM microscope. More details about this system can be found in [28].

\section{Results}

An example of SXR "water window" images of organic sample, acquired with the full-field SXR microscope is depicted in Fig. 2.
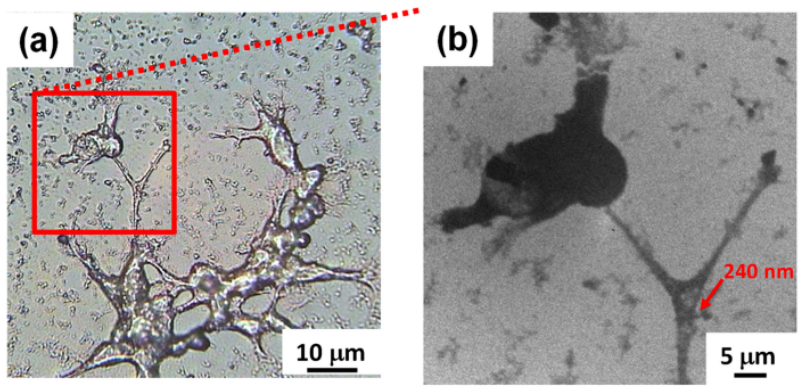

Fig. 2. CT 26 fibroblast cells. Comparison of optical image, 40x objective, (a) and detail imaged (b) with the SXR "water window" microscope (red square in (a)) that shows small features of the order of $240 \mathrm{~nm}$ (indicated with red arrow). Exposure of 200 EUV pulses.

Fig. 2a) and b) shows a sample of CT 26 fibroblast from Mus musculus colon carcinoma (strain BALB/c), prepared on top of a $30 \mathrm{~nm}$ thick $\mathrm{Si}_{3} \mathrm{~N}_{4}$ membrane. A direct comparison between the image acquired with a traditional optical microscope (Fig. 2a)) and the SXR microscope image (Fig. 2 b)), acquired with 200 SXR pulses, at a source repetition rate of $10 \mathrm{~Hz}$, exposure time of 22 seconds and detector (CCD) temperature of - 
$20{ }^{\circ} \mathrm{C}$, are shown. The sample was prepared with a gradual dehydration in ethanol series (final concentration $70 \%$ EtOH), without any fixation procedure. The SXR image shows improved spatial resolution due to the employment of shorter wavelength, beyond the diffraction limit of the optical-visible light microscopes. Some internal and external structures can be distinguished due to phase contrast in the visible light microscopy images and due to the modulation in the absorption of the SXR light through the sample in the SXR images.

The full field EUV microscope was employed for imaging of CT26 fibroblast cells. The EUV images were acquired with 200 EUV pulses - $22 \mathrm{sec}$. exposure time and detector temperature of $-20^{\circ} \mathrm{C}$. A sample of CT 26 cells fixed with $30 \%$ hexamethildisilazane (HDMS) in absolute $\mathrm{EtOH}$ on top of $30 \mathrm{~nm} \mathrm{Si}_{3} \mathrm{~N}_{4}$ membrane was imaged with the optical microscope, equipped with $40 \mathrm{x}$ objective, as depicted in Fig. 3a) and at $13.84 \mathrm{~nm}$ wavelength with the EUV microscope - Fig. 3b). It can be seen that the EUV image shows a very high contrast and resolution enhancement and permits to investigate features of the order of $100 \mathrm{~nm}$ or smaller in size. The EUV images exhibit superior spatial resolution and much higher optical magnification $(410 \mathrm{x})$ comparing to visible light microscopy - Fig. 3a). Moreover, although the EUV radiation does not allow to see the internal structure of the cells, it shows in detail the fine structures outside the cell membrane (interconnections between the cells, flagella, etc.), which are not visible in the SXR range due to the lack of optical contrast in those very thin features.
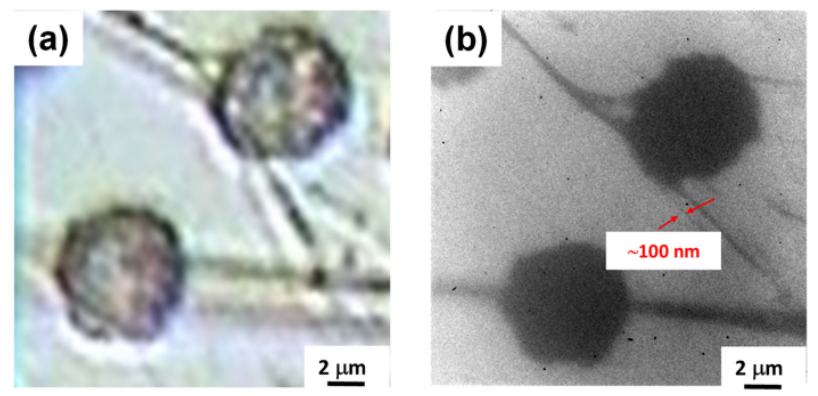

Fig. 3. Comparison of CT 26 fibroblast cells sample acquired with optical microscope (small section from the optical image acquired with 40x objective) (a) and EUV microscope (b). In this last image it is possible to appreciate small features of the order of $100 \mathrm{~nm}$ (as indicated in red arrows). Exposure of 200 EUV pulses.

The contact microscope has been used for imaging fixed epidermal cells (Keratinocytes), as depicted in Fig. 4. The sample was prepared in collaboration with the Institute of Biotechnology, Warsaw University of Technology, Poland. The sample was cultured on PMMA photoresist in a controlled culture conditions. A Dulbecco's Modified Eagle's Culture Medium (DMEM, Sigma-Aldrich) supplemented with 1\% L-glutamine, $1 \%$ penicillin/streptomycin and $10 \%$ heat inactivated foetal bovine serum (Celbio) was used as a culture medium. The culture medium was washed with PBS buffer solution followed by fixation and dehydration of the cells using paraformaldehyde $3.7 \%$ in phosphate buffered saline at $35{ }^{\circ} \mathrm{C}$. The exposure of 200 SXR pulses, 20 second exposure time, was required to expose the PMMA photoresist and reach spatial resolution of approximately $80 \mathrm{~nm}$ half-pitch.
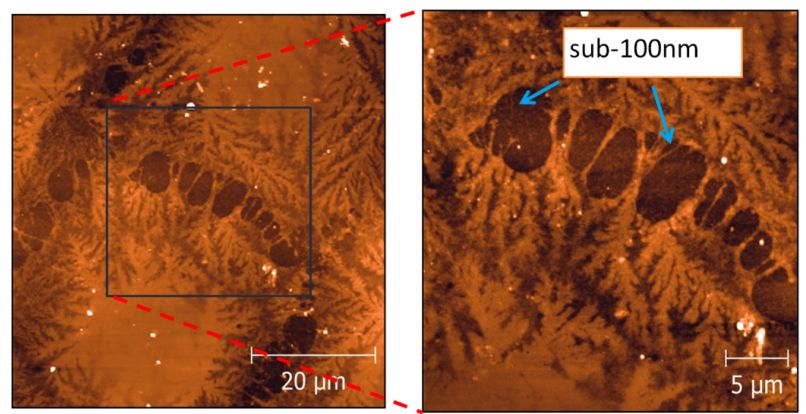

Fig. 4. SXR contact microscopy images of fixed epidermal cells (Keratinocytes). Images were obtained with SXR exposure of 200 pulses, 20 seconds and the photoresist was scanned using AFM microscope in semi-contact mode. Structures with sizes below $100 \mathrm{~nm}$ are visible.

The photoresist was treated with previously mentioned procedure and later scanned with AFM (AFM, NTMTD, Russia) operating in semi-contact mode. Each image was $512 \times 512$ points over $60 \times 60 \mu \mathrm{m}^{2}$ and $25 \times 25$ $\mu \mathrm{m}^{2}$ in left and right images, respectively. The structures with sizes below $100 \mathrm{~nm}$ are clearly visible. More details about this work can be found in [27]

\section{Summary and conclusions}

The SXR/EUV desk-top microscopes based on laser plasma sources with double stream gas puff target allow capturing magnified images of the samples, with 50-80 $\mathrm{nm}$ half-pitch spatial resolution and exposure time as low as few seconds. The microscopes are compact and permit to obtain images with a nanometer spatial resolution and short exposure time. Such characteristics represent key parameters which open possibility of a future commercialization of such systems. Those systems employ photons and not electrons, like in SEM, allow obtaining additional/complementary information about the sample. Their spatial resolution is comparable to what is possible to obtain with Stimulated Emission Depletion microscopy (STED) [29], however, such table-top systems allow for a direct acquisition of a full field images, in contrary to STED acquisition, operating in scanning mode and do not require fluorescent bio markers or staining that can modify the morphology of the sample. Additionally, short wavelengths provide high optical contrast, either in biological (SXR microscope) or in all matter (EUV microscope).

These microscopes may represent an important alternative to perform experiments in small academic laboratories and could have an impact on the nanotechnology in the near future. The goal of developing of these microscopes is to show feasibility to achieve high resolution imaging, low exposure times, 
together with a compact footprint, which may, in the future, open a possibility for commercialization.

This work is supported by the National Science Centre, Opus programmes, grant agreement number UMO2015/17/B/ST7/03718 and UMO-2015/19/B/ST3 /00435, the Education, Audiovisual and Culture Executive Agency (EACEA) Erasmus Mundus Joint Doctorate Programme Project No. 2012-0033 and from the European Union's Horizon 2020 research and innovation program, under Laserlab-Europe IV, grant agreement No. 654148. We would like to thank to Tomas Parkman, Šarka Salacová and Jana Turňová from Faculty of Biomedical Engineering, Czech Technical University in Prague for biological samples and Andrzej Kowalik and Mrs. Anna Rojek, from Institute of Electronic Materials Technology (ITME) for the preparation of the photoresist in their laboratory. We also acknowledge Dr. Tomasz Kobiela and Mrs. Anna Sobiepanek, from the Faculty of Chemistry Warsaw University of Technology for providing biological samples used for contact microscopy experiments.

\section{References}

1. The International Organization for Standardization, "ISO 21348 Definitions of Solar Irradiance Spectral Categories," 6-7 (2007).

2. R.N. Wilke, M. Priebe, M. Bartels, K. Giewekemeyer, A. Diaz, P. Karvinen, T. Salditt, Optics Express 20, 19232 (2012).

3. M. A. Le Gros, G. Mcdermott, B. P. Cinquin, Journal of Synchrotron Radiation 21 (6), 1-8 (2014).

4. G. Schneider, Ultramicroscopy 75, 85-104 (1998)

5. K.W. Kim, Y. Kwon, K. Nam, J. Lim, K.W. Kim, K. Chon, B. Kim, D.E. Kim, J. Kim, B.N. Ahn, H.J. Shin, S. Rah, K.W. Kim, J.S. Chae, D.G. Gweon, D.W. Kang, S.H. Kang, J.Y. Min, K. Choi, S.E. Yoon, E. Kim, Y. Namba, K. Yoon, Physics in Medicine and Biology 51, N99-N107 (2006)

6. H. T. Kim, Springer Proceedings in Physics 136, 323-328 (2010)

7. M. Dierolf, New J. Phys. 12, 035017 (2010)

8. P. W. Wachulak, R. A. Bartels, M. C. Marconi, C. S. Menoni, J.J. Rocca, Y. Lu, B. Parkinson, Optics Express 14, 9636 (2006)

9. M.C. Marconi, P.W. Wachulak, Progress in Quantum Electronics 34, 173-190 (2010)

10. P.W. Wachulak, L. Wegrzynski, A. Bartnik, T. Fok, R. Jarocki, J. Kostecki, M. Szczurek and H. Fiedorowicz, Laser and Particle Beams 31, 2, 219227 (2013)

11. C. Brewer, F. Brizuela, P. Wachulak, D.H. Martz, W. Chao, E.H. Anderson, D.T. Attwood, A. V Vinogradov, I. a Artyukov, A.G. Ponomareko, V. V Kondratenko, M.C. Marconi, J.J. Rocca, C.S. Menoni, Optics Letters 33, 518-520 (2008)

12. P.W. Wachulak, C.A. Brewer, F. Brizuela, W. Chao, E. Anderson, R. A. Bartels, C.S. Menoni, J.J. Rocca, M.C. Marconi , Journal of the Optical Society of America B 25, B20, (2008)
13. E. B. Malm, N. C. Monserud, C. G. Brown, P. W. Wachulak, H. Xu, G. Balakrishnan, W. Chao, E. Anderson, M. C. Marconi, Optics Express21, 8, 9959-9966 (2013)

14. P.W. Wachulak, M.C. Marconi, R. a Bartels, C.S. Menoni, J.J. Rocca, Journal of the Optical Society of America B 25, 1811-1814 (2008)

15. G. Vaschenko, F. Brizuela, C. Brewer, M. Grisham, H. Mancini, C.S. Menoni, M.C. Marconi, J.J. Rocca, W. Chao, J. Liddle, E.H. Anderson, D.T. Attwood, V Vinogradov, I. Artioukov, Y.P. Pershyn, V. V Kondratenko, Optics Letters 30, 2095-7 (2005)

16. L. Juschkin, Journal of Physics: Conference Series 186, 012030 (2009)

17. H. Legall, G. Blobel, H. Stiel, W. Sandner, C. Seim, P. Takman, D. H. Martz, M. Selin, U. Vogt, H. M. Hertz, D. Esser, H. Sipma, J. Luttmann, M. Höfer, H. D. Hoffmann, S. Yulin, T. Feigl, S. Rehbein, P. Guttmann, G. Schneider, U. Wiesemann, M. Wirtz, and W. Diete, Optics Express 20, 16, 18362 (2012)

18. J. Kirz, Rev. Sci. Instr. 56, 1-13 (1985)

19. P. C. Cheng, NIMA 246, 668-674 (1986)

20. M. Kado, M. Kishimoto, S. Tamotsu, K. Yasuda, M. Aoyama and K. Shinohara, Proc. of SPIE 8849, 88490C-1 (2013)

21. A. Bartnik, W. Lisowski, J. Sobczak, P. Wachulak, B. Budner, B. Korczyc, H. Fiedorowicz, Appl Phys A 109, 39-43 (2012)

22. P. W. Wachulak, A. Bartnik, M. Skorupka, J. Kostecki, R. Jarocki, M. Szczurek, L. Wegrzynski, T. Fok and H. Fiedorowicz, Applied Physics B 111, 2, 239-247 (2013),

23. J.M. Heck, D.T. Attwood, W. Meyer-Ilse, E.H. Anderson, J. X-ray Sci. Technol. 8, 95 (1998)

24. P. Wachulak, A. Torrisi, M.F. Nawaz, A. Bartnik, D. Adjei, Š. Vondrová, J. Turňová, A. Jančarek, J. Limpouch, M. Vrbová, H. Fiedorowicz, Microscopy and Microanalysis 21, 1214-1223 (2015)

25. P. W. Wachulak, A. Torrisi, A. Bartnik, D. Adjei, J. Kostecki, L. Wegrzynski, R. Jarocki, M. Szczurek, H. Fiedorowicz, Applied Physics B 118, 573-578 (2015)

26. P. W. Wachulak, A. Torrisi, A. Bartnik, Ł. Węgrzyński, T. Fok and H. Fiedorowicz, Applied Physics B 123:25, 1-5 (2017),

27. A. Torrisi, P. Wachulak, Ł. Węgrzyński, T. Fok, A. Bartnik, T. Parkman, Š. Vondrová, J. Turňová, B. J. Jankiewicz, B. Bartosewicz and H. Fiedorowicz, Journal of Microscopy 265, 2, 251-260 (2017),

28. M.G. Ayele, J. Czwartos, D. Adjei, P. Wachulak, I.U. Ahad, A. Bartnik, Ł. Wegrzynski, M. Szczurek, R. Jarocki, H. Fiedorowicz, M. Lekka, K. Pogoda and J. Gostek, Acta Physica Polonica 129, 2, 237240 (2016),

29. K. Otomo, T. Hibi, Y. Kozawa T. Nemoto, Microscopy (Oxf) 64, 4, 227-36 (2015) 